\title{
A new image processing heuristic suggested by optimization experiments - Enhance it before you Lose it
}

\author{
Rajeswari Raju ${ }^{\mathrm{a}, \text {, },}$, Tomas Maul ${ }^{\mathrm{a}}$, Andrzej Bargiela ${ }^{\mathrm{b}}$ \\ ${ }^{a}$ University of Nottingham, Faculty of Science, School of Computer Science, Malaysia Campus, Semenyih, 43500 \\ Selangor, Malaysia \\ ${ }^{\mathrm{b}}$ University of Nottingham, Faculty of Science, School of Computer Science, ,Jubilee Campus, Nottingham, \\ NG81BB,United Kingdom \\ ${ }^{\mathrm{c} U n i v e r s i t y ~ T e c h n o l o g y ~ M A R A, ~ F a c u l t y ~ o f ~ C o m p u t e r ~ a n d ~ M a t h e m a t i c a l ~ S c i e n c e, ~ M a l a y s i a ~}$ \\ *Corresponding Author: khyx1rru@ nottingham.edu.my
}

\begin{abstract}
In this paper we report an interesting observation pertaining to Denoising based on the optimization of image processing chains. Although often a goal in itself, Denoising is usually performed in order to minimize the detrimental effects of noise in the subsequent stages of an algorithm. Typically, Denoising is carried out as an early pre-processing stage before other core functions are applied. In the context of optimizing image processing chains for membrane detection, we gathered statistics pertaining to 30 'good' chains, all of which exhibited an average F1 score larger than $90 \%$, and observed that not one was found to use a 'Denoising function' as its $1^{\text {st }}$ step in the processing chain. On the contrary, the optimization process tended to choose Denoising as a middle processing component, and generally selected image enhancement as an earlier component. We conclude, that at least in the context of this membrane detection problem, it is better to enhance information before cleaning (or losing) it.
\end{abstract}

Keywords: membrane detection, denoising, segmentation, image processing, optimization.

\section{Introduction}

One of the aims of our research is to identify the best possible sequence of image processing functions; capable of efficiently and accurately detecting neuronal membranes whilst ignoring and/or removing extraneous organelles from the processed output ${ }^{(1)}$. The problem of membrane detection, which can be seen to belong to the general class of segmentation problems, is characterized by several issues, including over and under segmentation due to similarities between membrane and non-membrane material. Many algorithms depend on ground-truth for training and require large numbers of labelled training samples which is expensive and generally involves several time consuming processes $^{(1)}$. In order to detect membranes whilst eliminating extraneous organelles we have proposed an approach called Image Processing Chain Optimization (IPCO). This approach (1) attains competitive accuracy levels whilst not requiring an excessively long tuning phase, (2) does not require specialized hardware, (3) leads to chains consisting of short sequences of basic processing steps which are efficient and easy to interpret ${ }^{(1)}$, (4) is simple to use ${ }^{(1)}$ and (5) is flexible and can be applied to many different types of datasets. In carrying out our experiments we have discovered several interesting facts about optimal image processing chains, some of which are presented in this paper.

\section{Background Study}

According to Rafael C.Gonzales et.al ${ }^{(2)}$, image analysis is a research area lying somewhere in between image processing and computer vision. According to this there are three types of processing, distinguished by different levels of abstraction, which are: low-level; mid-level; and high-level. Low-level processing involves: image preprocessing to reduce noise, contrast enhancement, and image sharpening. Mid-level processing involves: image 
segmentation, description of objects in a form suitable for further computational processing and classification or recognition of individual objects. Finally, high-level processing involves: making sense of an ensemble of recognized objects and performing cognitive functions associated with human vision. In this paper we focus on a crucial low-level component, which is Denoising, in the context of serial section Transmission Electron Microscopy (ssTEM). Denoising plays an important role in many image based applications, such as: image restoration; visual tracking; image registration; image segmentation and image classification. In the sSTEM context, noise can be seen to consist of two main parts: the quantum noise of the electron beam and the noise originating from the image recording system $^{(3)}$. In general, the existence of noise causes images to get a mottled, grainy, textured or snowy appearance ${ }^{(4)}$. So in order to minimize the noise, we need to adopt a Denoising technique which leads to a better output for human and/or computer inspection. Many methods, regardless of specific problem domain, share the same issue whereby although noise reduction can improve performance it can also cause information loss, for example through blurring ${ }^{(5)}$. Image Denoising algorithms are arguably one of the oldest image processing functions. In spite of this, researchers continue to focus their attention on it in order further improve the state-of-the-art ${ }^{(6)}$.

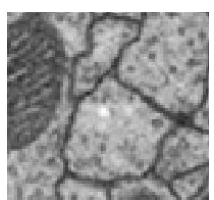

Original Image

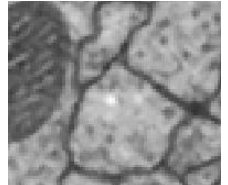

After Denoise-Filter
Fig. 1. Image Before and After Denosing

\subsection{How is Image Processing typically done?}

By default, before we process an image, it is considered good practice to filter out any 'noise'. Until today image noise suppression remains a challenge in the area of image processing, especially when the images are acquired under poor visibility conditions and therefore noise levels are $\operatorname{high}^{(7)}$. Typically, the Denoising function estimates the "original image" by eliminating noise from a noise-contaminated version of the image ${ }^{(7)}$, where noise can be caused by internal or external (environmental) conditions. More importantly for this paper's main argument, Denoising or noise filtering, is typically considered a first (or early) step in the preprocessing stages of an image based application. Image Denoising is a common preprocessing step in many Magnetic Resonance (MR) image processing and analysis tasks, such as segmentation ${ }^{(8)}$, registration ${ }^{(9)}$ or parametric image synthesis ${ }^{(10)}$. Denoising is considered as one of the core challenges in image preprocessing, and effective Denoising is often crucial for subsequent components to be able to carry out their functions adequately. According to S. Annadurai and R. Shanmugalakshmi preprocessing is a process to condition or enhance an image in order to make it suitable for further processing ${ }^{(11)}$.

\subsection{Our suggestion - How is Image Processing supposed to be done?}

According to Kenneth R. Spring et.al, the first step in image processing is to remove brightness fluctuations (due to uneven background illumination). Then this step should be followed by removing the noise introduced by the specimen or camera system ${ }^{(12)}$. A. Buedes et.al stated that noise reduction is imperative, and it should be done in the correct way and with the right workflow ${ }^{(13)}$. According to the authors ${ }^{(13)}$, by applying the noise reduction without proper evaluation, this will not only eliminate noise but will also remove fine details that may be necessary at later stages in the workflow. This suggests that we should not always remove noise as a first step in the workflow. Moreover, we should consider the possibility of retaining and enhancing different details at different stages, and likewise we should consider suppressing different aspects of noise, at different stages, according to the application under consideration. Denoised images may appear good qualitatively and yet may be considered poor from a more quantitate perspective, and especially considering specific applications. For example, in the fields of medical imaging ${ }^{(14)}$, a minor distortion (as perceived subjectively) may play a big role in terms of the scientific enquiry it informs. Medical imaging acquisition technologies produce different types of noise and artifacts in the images they generate ${ }^{(12)}$, which should be treated using an appropriate workflow, since in medical imaging, every detail of information is important. The Denoising process should not damage any anatomical details pertinent to the clinical (e.g. diagnostic) aims ${ }^{(12,15)}$.

In conclusion, the common practice is for Denoising to be 
done early, but the literature shows some applications where details need to be protected in the early stages and where noise is not always filtered out as first step in an image processing sequence. In our findings (Section 2.3), we encounter that Denoising is being done after enhancement (often even after classification), and this tends to give better F1 scores on average (more than 91\%).

\subsection{Our Findings}

When carrying our research in membrane detection and organelle elimination, where activities ranged from manual fine tuning ${ }^{(1)}$ to automated segmentation using IPCO, we found that, at least for this membrane detection problem, Denoising typically appears later in the sequence (or chain) of processing functions. Moreover, in 10 cases out of 10 (including the best chain), we find contrast enhancement before Denoising, suggesting that details need to be enhanced before cleaned, which could be encapsulated by the heuristic "enhance it before you lose it". In many cases even classification is done before Denoising.

The following section briefly summarizes the software tools used, our chosen dataset and the algorithmic approach adopted. This is followed by the results section which includes an overview of the optimal chains obtained, followed by a brief conclusion.

\section{Methodology}

\section{1 Image Processing Platform - MatLab and the Image Processing Toolbox}

Our approach is based on a sequence of basic image processing steps, most of which we adopted from MatLab's image processing toolbox by MathWorks. This toolbox is useful for the processing, visualization and analysis of images, whilst MatLab is convenient for rapid prototyping.

\subsection{Data}

The experiments were performed on data provided by the ISBI 2012 (IEEE International Symposium on Biomedical Imaging) challenge: "Segmentation of neuronal structures in Electron Microscopy (EM) stacks"(16). Albert Cardona and team provide public access to 30 slices of Transmission Electron Microscopy (TEM) images with corresponding ground-truth images for training, and a second set of 30 TEM images for testing ${ }^{(17)}$.

\subsection{Image Processing Chain Optimization (IPCO) methods}

IPCO consist of chains or simple networks of image processing functions optimized via a global stochastic algorithm, which combines elements of genetic algorithms and differential evolution. The process optimizes the relative position of functions and several parameters intrinsic to each function (e.g. window radii and thresholds). IPCO works with a single linear chain of functions. For the experiments reported in this paper that maximum number of functions allowed per chain was set to 8. Using IPCO, we ran optimizations to a target cost of 0 or for a maximum of 10000 generations, whichever one occurred first.

\section{Experimental Result}

\subsection{IPCO Functions}

IPCO can lead to a diverse set of useful chains or networks many of which consist of unorthodox sequences and choices of functions.

Table 1. Main classes of IPCO functions with their corresponding image processing phases and general purposes

\begin{tabular}{|l|l|l|}
\hline $\begin{array}{l}\text { Choices of Processing } \\
\text { Functions }\end{array}$ & $\begin{array}{l}\text { Image } \\
\text { processing } \\
\text { Phase }\end{array}$ & $\begin{array}{l}\text { Functions } \\
\text { General } \\
\text { Purpose }\end{array}$ \\
\hline Denoising & Pre-processing & Cleaning \\
\hline Contrast Enhancement & Pre-processing & Enhancing \\
\hline Thresholding & Classification & Classifying \\
\hline Hole Filling & Classification & Classifying \\
\hline Watershed & Classification & Classifying \\
\hline $\begin{array}{l}\text { Combination Function } \\
\text { of MinMax, Average } \\
\text { and Multiply }\end{array}$ & Classification & Hybrid \\
\hline $\begin{array}{l}\text { Morphological } \\
\text { Operators }\end{array}$ & Post-processing & Cleaning \\
\hline
\end{tabular}

Table 1 shows the main classes of IPCO functions with their corresponding image processing phases. The proposed Image Processing Chain Optimization 
approach uses global stochastic optimization to optimize chains of simple image processing functions. IPCO consists of several pre-processing functions (i.e. several types of Denoising and Contrast Enhancement), classification functions (i.e. Thresholding, Hole-filling and Watershed) and post-processing functions (i.e. several types of Morphological Operators). Each processing step has its own parameters which require some data-dependent fine-tuning. Several manual fine-tuning experiments were carried out, in order to find the most favourable set of parameters in terms of accuracy (i.e. F1 score) and speed. The end result of IPCO processing is an image where pixels that are classified as "membrane" are labelled as "1" whereas pixels classified as "non-membrane" are labelled as "0". The 0-labelled pixels include various organelles that are eliminated from the image. Such a binary 0-1 image is compared with the binary image of the ground truth to identify pixels that are identified correctly and incorrectly. Pixels that are falsely identified as membranes in the IPCO output but are classed as non-membrane pixels in the ground truth image correspond to false positives. Conversely pixels that are identified as non-membrane by IPCO but are classed as membrane in the ground truth image are referred to as false negatives.

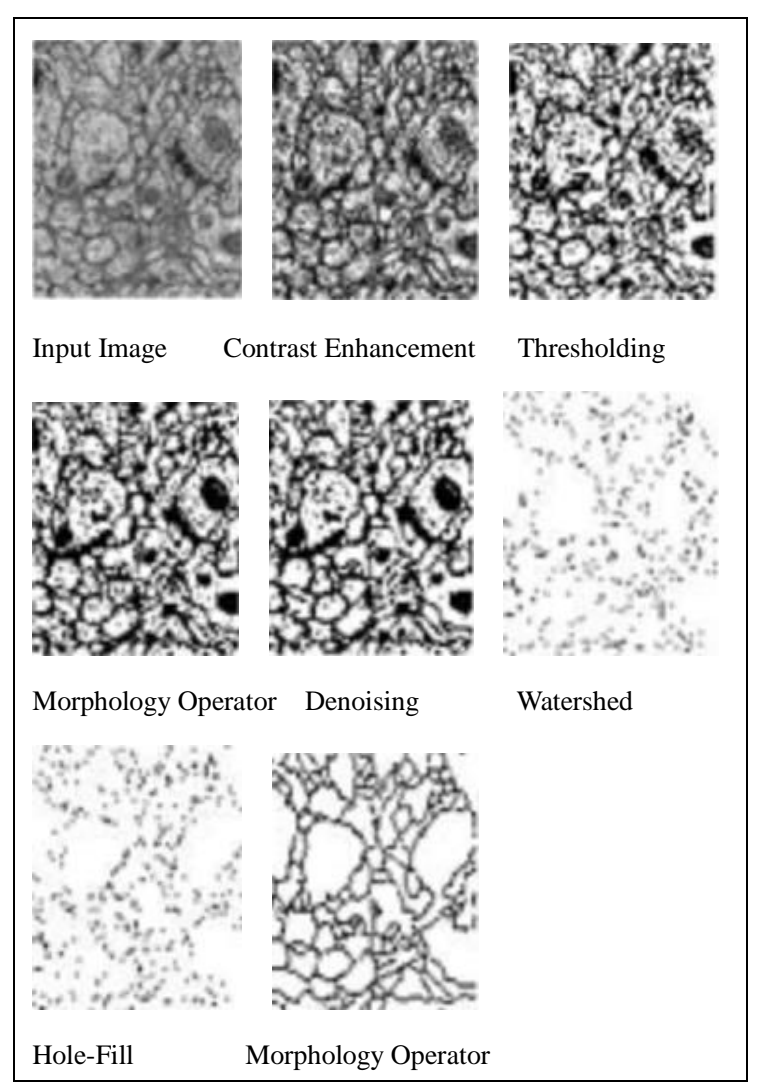

Fig. 2. Sequence of functions and processed images of chain with an F1 score of $91.67 \%$
Fig. 2 shows the functions and initial, intermediate and final processed images of an IPCO chain with an F1 score of $91.67 \%$. From the Fig.2, we can see that the Median Denoising function appears in the middle of the processing chain (i.e. stage 4).

\subsection{Experimental results pertaining to Denoising}

In what relative position in a chain does the Denoising function typically fall? According to the results depicted in Table 3, the Denoising function can be found in second place in $50 \%$ of good chains, third place in $20 \%$ of chains, fourth place in $40 \%$ of chains, fifth place in $20 \%$ of chains, sixth place in $10 \%$ of chain, and in $8^{\text {th }}$ place again in $10 \%$ of chains. Note that for Table 3, our definition of a "good chain" is one with an F1 score larger than $91 \%$.

Below in Table 2 we depict IPCO processing chains with an F1 score larger than $91 \%$, averaged over all training images. These chains are a subset of the chains depicted in Table 3. We also indicate function types (e.g. Denoising) and sub-type (e.g. Median). Below the table is a legend describing the abbreviations used. The table shows that for chains with an average (Avg) F1 score larger than 91\%, the optimizer tends to choose the Median function for Denoising and the Adapthisteq (Local Contrast Enhancement) function for Contrast Enhancement.

Table 2. IPCO processing chains with an F1 score larger than $91 \%$, averaged over all training images

\begin{tabular}{|c|c|c|c|c|c|c|c|c|c|c|}
\hline \multirow{2}{*}{$\begin{array}{l}\text { Denoisi } \\
\text { ng Place }\end{array}$} & \multirow{2}{*}{$\begin{array}{l}\text { Iterati } \\
\text { on } \\
\text { Count }\end{array}$} & \multirow{2}{*}{$\begin{array}{l}\text { Scores } \\
\text { (Avg } \\
\text { F1) }\end{array}$} & \multicolumn{3}{|c|}{$\begin{array}{l}\text { Functions } \\
\text { positions }\end{array}$} & \multicolumn{3}{|c|}{ and their } & \multicolumn{2}{|c|}{ chain } \\
\hline & & & 1 & 2 & 3 & 4 & 5 & 6 & 7 & 8 \\
\hline 4th & 3414 & 91.67 & $\begin{array}{l}\mathrm{C} \\
\mathrm{A}\end{array}$ & $\begin{array}{l}\mathrm{T} \\
\mathrm{S}\end{array}$ & $\begin{array}{l}\mathrm{M} \\
\mathrm{O} \\
\mathrm{O}\end{array}$ & $\begin{array}{l}\text { D } \\
\text { M }\end{array}$ & W & $\mathrm{H}$ & $\begin{array}{l}\text { M } \\
\text { O } \\
\text { E }\end{array}$ & \\
\hline 4th & 200 & 91.64 & $\begin{array}{l}\mathrm{C} \\
\mathrm{A}\end{array}$ & $\begin{array}{l}\mathrm{T} \\
\mathrm{S}\end{array}$ & $\mathrm{H}$ & $\begin{array}{l}\text { D } \\
\text { M }\end{array}$ & $\begin{array}{l}\mathrm{M} \\
\mathrm{O} \\
\mathrm{O}\end{array}$ & & & \\
\hline $\begin{array}{l}2^{\text {nd }} \text { and } \\
3 \text { rd }\end{array}$ & 96 & 91.43 & $\begin{array}{l}\mathrm{C} \\
\mathrm{A}\end{array}$ & $\begin{array}{l}\text { D } \\
\text { M }\end{array}$ & $\begin{array}{l}\text { D } \\
\text { M }\end{array}$ & $\begin{array}{l}\mathrm{C} \\
\mathrm{M} \\
\mathrm{M}\end{array}$ & $\begin{array}{l}\mathrm{T} \\
\mathrm{S}\end{array}$ & $\mathrm{H}$ & & \\
\hline $\begin{array}{l}2^{\text {nd }} 4^{\text {th }} \\
\text { and } 8 \text { th }\end{array}$ & 7003 & 91.35 & $\begin{array}{l}\mathrm{C} \\
\mathrm{A}\end{array}$ & $\begin{array}{l}\text { D } \\
\text { M }\end{array}$ & $\mathrm{H}$ & $\begin{array}{l}\text { D } \\
\text { M }\end{array}$ & $\begin{array}{l}\mathrm{C} \\
\mathrm{M} \\
\mathrm{M}\end{array}$ & $\begin{array}{l}\mathrm{T} \\
\mathrm{S}\end{array}$ & $\mathrm{H}$ & $\begin{array}{l}\mathbf{D} \\
\mathbf{M}\end{array}$ \\
\hline 5 th & 487 & 91.27 & $\mathrm{C}$ & $\mathrm{C}$ & TS & $\mathrm{H}$ & D & & & \\
\hline
\end{tabular}




\begin{tabular}{|c|c|c|c|c|c|c|c|c|c|}
\hline & & & A & $\begin{array}{l}\mathrm{O} \\
\mathrm{A}\end{array}$ & & & M & & \\
\hline 6th & 200 & 91.15 & $\begin{array}{l}\mathrm{C} \\
\mathrm{A}\end{array}$ & $\begin{array}{l}\mathrm{T} \\
\mathrm{S}\end{array}$ & $\mathrm{H}$ & $\begin{array}{l}\mathrm{T} \\
\mathrm{S}\end{array}$ & $\begin{array}{l}\mathrm{M} \\
\mathrm{O} \\
\mathrm{O}\end{array}$ & $\begin{array}{l}\text { D } \\
\text { M }\end{array}$ & $\begin{array}{l}\mathrm{M} \\
\mathrm{O} \\
\mathrm{O}\end{array}$ \\
\hline $\begin{array}{ll}2^{\text {nd }} \text { and } \\
4 \text { th }\end{array}$ & 2548 & 91.12 & $\begin{array}{l}\mathrm{C} \\
\mathrm{A}\end{array}$ & $\begin{array}{l}\mathbf{D} \\
\mathbf{M}\end{array}$ & $\begin{array}{l}\mathrm{C} \\
\mathrm{M} \\
\mathrm{M}\end{array}$ & $\begin{array}{l}\text { D } \\
\text { M }\end{array}$ & $\begin{array}{l}\mathrm{T} \\
\mathrm{S}\end{array}$ & $\mathrm{H}$ & \\
\hline 2nd & 200 & 91.11 & $\begin{array}{l}\mathrm{C} \\
\mathrm{N} \\
\end{array}$ & $\begin{array}{l}\mathbf{D} \\
\mathbf{M} \\
\end{array}$ & $\mathrm{H}$ & $\begin{array}{l}\mathrm{T} \\
\mathrm{S} \\
\end{array}$ & & & \\
\hline 2nd & 70 & 91.01 & $\begin{array}{l}\mathrm{C} \\
\mathrm{A} \\
\end{array}$ & $\begin{array}{l}\mathbf{D} \\
\mathbf{M}\end{array}$ & $\mathrm{TS}$ & $\begin{array}{l}\mathrm{T} \\
\mathrm{S}\end{array}$ & $\mathrm{H}$ & & \\
\hline $\begin{array}{l}3^{\text {rd }} \text { and } \\
5 \text { th }\end{array}$ & 324 & 91.00 & $\begin{array}{l}\mathrm{C} \\
\mathrm{A}\end{array}$ & $\begin{array}{l}\mathrm{T} \\
\mathrm{S}\end{array}$ & $\begin{array}{l}\text { D } \\
\text { M }\end{array}$ & $\mathrm{H}$ & $\begin{array}{l}\text { D } \\
\text { M }\end{array}$ & $\begin{array}{l}\mathrm{C} \\
\mathrm{O} \\
\mathrm{A}\end{array}$ & $\begin{array}{l}\mathrm{T} \\
\mathrm{S}\end{array}$ \\
\hline
\end{tabular}

Legends

\begin{tabular}{|l|l|l|}
\hline CA - Contrast & H - Hole Filling & $\begin{array}{l}\text { COM - } \\
\text { Enhancement }\end{array}$ \\
Adapthisteq & W - Watershed & $\begin{array}{l}\text { Multiply } \\
\text { CN - Contrast }\end{array}$ \\
Enhancement NBins & Operator Eroding & $\begin{array}{l}\text { CMM - } \\
\text { Combine }\end{array}$ \\
TS - Thresholding & MOO - Morphological & MinMax \\
Simple & Operator Opening & COA - Combine \\
DM - Denoise Median & & Average \\
\hline
\end{tabular}

Table 3. IPCO processing chains with an F1 score larger than $90 \%$, averaged over all training images

\begin{tabular}{|c|c|c|c|c|c|c|c|c|c|}
\hline \multirow{2}{*}{$\begin{array}{l}\text { Denoisi } \\
\text { ng } \\
\text { Place }\end{array}$} & \multirow{2}{*}{$\begin{array}{l}\text { Scores } \\
\text { (Avg } \\
\text { F1) }\end{array}$} & \multicolumn{8}{|c|}{ Functions and their chain positions } \\
\hline & & 1 & 2 & 3 & 4 & 5 & 6 & 7 & 8 \\
\hline $\begin{array}{l}\text { 2nd } \\
\text { 4th, and } \\
\text { 8th }\end{array}$ & 91.35 & $\begin{array}{l}\mathrm{C} \\
\mathrm{A}\end{array}$ & $\begin{array}{l}\mathbf{D} \\
\mathbf{M}\end{array}$ & $\mathrm{H}$ & $\begin{array}{l}\text { D } \\
\text { M }\end{array}$ & $\begin{array}{l}\mathrm{C} \\
\mathrm{M} \\
\mathrm{M}\end{array}$ & $\begin{array}{l}\mathrm{T} \\
\mathrm{S}\end{array}$ & $\mathrm{H}$ & DM \\
\hline $\begin{array}{l}3^{\text {rd }} \text { and } \\
8 \text { th }\end{array}$ & 90.98 & $\mathrm{H}$ & $\begin{array}{l}\mathrm{C} \\
\mathrm{M} \\
\mathrm{M}\end{array}$ & $\begin{array}{l}\text { D } \\
\text { M }\end{array}$ & $\begin{array}{l}\text { C } \\
\text { A }\end{array}$ & $\mathrm{H}$ & $\begin{array}{l}\mathrm{C} \\
\mathrm{O} \\
\mathrm{A}\end{array}$ & $\begin{array}{l}\mathrm{T} \\
\mathrm{S}\end{array}$ & DM \\
\hline 5 th & 90.58 & $\begin{array}{l}\mathrm{T} \\
\mathrm{S}\end{array}$ & $\begin{array}{l}\mathrm{M} \\
\mathrm{O} \\
\mathrm{O}\end{array}$ & W & $\begin{array}{l}\mathrm{M} \\
\mathrm{O} \\
\mathrm{E}\end{array}$ & $\begin{array}{l}\text { D } \\
\text { M }\end{array}$ & $\begin{array}{l}\mathrm{C} \\
\mathrm{I}\end{array}$ & $\begin{array}{l}\mathrm{C} \\
\mathrm{I}\end{array}$ & CI \\
\hline 2nd & 90.40 & $\begin{array}{l}\mathrm{C} \\
\mathrm{A}\end{array}$ & $\begin{array}{l}\mathbf{D} \\
\mathbf{M}\end{array}$ & $\begin{array}{l}\mathrm{T} \\
\mathrm{D}\end{array}$ & $\begin{array}{l}\text { C } \\
\text { A }\end{array}$ & $\mathrm{W}$ & $\begin{array}{l}\mathrm{T} \\
\mathrm{S}\end{array}$ & $\begin{array}{l}\mathrm{M} \\
\mathrm{O} \\
\mathrm{E}\end{array}$ & CMM \\
\hline 6 th & 90.00 & $\mathrm{H}$ & $\begin{array}{l}\mathrm{C} \\
\mathrm{H}\end{array}$ & $\begin{array}{l}\mathrm{C} \\
\mathrm{H}\end{array}$ & $\begin{array}{l}\mathrm{T} \\
\mathrm{D}\end{array}$ & $\begin{array}{l}\mathrm{T} \\
\mathrm{D}\end{array}$ & $\begin{array}{l}\mathbf{D} \\
\mathbf{M}\end{array}$ & $\begin{array}{l}\mathrm{C} \\
\mathrm{O}\end{array}$ & TS \\
\hline
\end{tabular}

\begin{tabular}{|c|c|c|c|c|c|c|c|c|}
\hline & & & & & & & & A \\
\hline 4th & 91.67 & $\begin{array}{l}\mathrm{C} \\
\mathrm{A}\end{array}$ & $\begin{array}{l}\mathrm{T} \\
\mathrm{S}\end{array}$ & $\begin{array}{l}\mathrm{M} \\
\mathrm{O} \\
\mathrm{O}\end{array}$ & $\begin{array}{l}\text { D } \\
\text { M }\end{array}$ & W & $\mathrm{H}$ & $\begin{array}{l}\mathrm{M} \\
\mathrm{O}\end{array}$ \\
\hline $\begin{array}{l}3^{\text {rd }} \text { and } \\
5 \text { th }\end{array}$ & 91.34 & $\begin{array}{l}\mathrm{C} \\
\mathrm{A}\end{array}$ & $\begin{array}{l}\mathrm{T} \\
\mathrm{S}\end{array}$ & $\begin{array}{l}\mathbf{D} \\
\mathbf{M}\end{array}$ & $\mathrm{H}$ & $\begin{array}{l}\text { D } \\
\text { M }\end{array}$ & $\begin{array}{l}\mathrm{C} \\
\mathrm{O} \\
\mathrm{A}\end{array}$ & $\begin{array}{l}\mathrm{T} \\
\mathrm{S}\end{array}$ \\
\hline 6th & 91.15 & $\begin{array}{l}\mathrm{C} \\
\mathrm{A}\end{array}$ & $\begin{array}{l}\mathrm{T} \\
\mathrm{S}\end{array}$ & $\mathrm{H}$ & $\begin{array}{l}\mathrm{T} \\
\mathrm{S}\end{array}$ & $\begin{array}{l}\mathrm{M} \\
\mathrm{O} \\
\mathrm{O}\end{array}$ & $\begin{array}{l}\mathbf{D} \\
\mathbf{M}\end{array}$ & $\begin{array}{l}\mathrm{M} \\
\mathrm{O} \\
\mathrm{O}\end{array}$ \\
\hline $\begin{array}{l}\text { 2nd and } \\
6 \text { th }\end{array}$ & 90.37 & $\mathrm{H}$ & $\begin{array}{l}\text { D } \\
\text { M }\end{array}$ & $\begin{array}{l}\mathrm{T} \\
\mathrm{D}\end{array}$ & $\begin{array}{l}\mathrm{C} \\
\mathrm{C}\end{array}$ & $\begin{array}{l}\mathrm{C} \\
\mathrm{M} \\
\mathrm{M}\end{array}$ & $\begin{array}{l}\mathbf{D} \\
\mathbf{W}\end{array}$ & $\begin{array}{l}\mathrm{M} \\
\mathrm{O} \\
\mathrm{O}\end{array}$ \\
\hline $\begin{array}{l}\text { 2nd and } \\
6 \text { th }\end{array}$ & 90.15 & $\mathrm{H}$ & $\begin{array}{l}\text { D } \\
\text { M }\end{array}$ & $\mathrm{H}$ & $\begin{array}{l}\mathrm{C} \\
\mathrm{A}\end{array}$ & $\begin{array}{l}\mathrm{T} \\
\mathrm{S}\end{array}$ & $\begin{array}{l}\mathbf{D} \\
\mathbf{M}\end{array}$ & $\begin{array}{l}\mathrm{T} \\
\mathrm{S}\end{array}$ \\
\hline 6th & 90.10 & $\begin{array}{l}\mathrm{C} \\
\mathrm{I}\end{array}$ & $\begin{array}{l}\mathbf{T} \\
\mathbf{S}\end{array}$ & $\begin{array}{l}\mathrm{T} \\
\mathrm{S}\end{array}$ & $\mathrm{H}$ & $\begin{array}{l}\mathrm{C} \\
\mathrm{I}\end{array}$ & $\begin{array}{l}\mathbf{D} \\
\mathbf{M}\end{array}$ & $\mathrm{H}$ \\
\hline $\begin{array}{l}\text { 2nd and } \\
5 \text { th }\end{array}$ & 90.02 & $\begin{array}{l}\mathrm{C} \\
\mathrm{A}\end{array}$ & $\begin{array}{l}\text { D } \\
\text { M }\end{array}$ & $\mathrm{H}$ & $\begin{array}{l}\mathrm{C} \\
\mathrm{M} \\
\mathrm{M}\end{array}$ & $\begin{array}{l}\text { D } \\
\text { M }\end{array}$ & $\mathrm{H}$ & $\begin{array}{l}\mathrm{T} \\
\mathrm{S}\end{array}$ \\
\hline $\begin{array}{l}\text { 2nd and } \\
\text { 3rd }\end{array}$ & 91.43 & $\begin{array}{l}\mathrm{C} \\
\mathrm{A}\end{array}$ & $\begin{array}{l}\text { D } \\
\text { M }\end{array}$ & $\begin{array}{l}\text { D } \\
\mathbf{M}\end{array}$ & $\begin{array}{l}\mathrm{C} \\
\mathrm{M} \\
\mathrm{M}\end{array}$ & $\begin{array}{l}\mathrm{T} \\
\mathrm{S}\end{array}$ & $\mathrm{H}$ & \\
\hline 5 th & 91.12 & $\begin{array}{l}\mathrm{C} \\
\mathrm{A}\end{array}$ & $\mathrm{H}$ & $\mathrm{H}$ & $\begin{array}{l}\mathrm{T} \\
\mathrm{S}\end{array}$ & $\begin{array}{l}\text { D } \\
\text { M }\end{array}$ & $\begin{array}{l}\mathrm{T} \\
\mathrm{S}\end{array}$ & \\
\hline $\begin{array}{l}\text { 2nd and } \\
4 \text { th }\end{array}$ & 91.12 & $\begin{array}{l}\mathrm{C} \\
\mathrm{A}\end{array}$ & $\begin{array}{l}\text { D } \\
\text { M }\end{array}$ & $\begin{array}{l}\mathrm{C} \\
\mathrm{M} \\
\mathrm{M}\end{array}$ & $\begin{array}{l}\text { D } \\
\mathbf{M}\end{array}$ & $\begin{array}{l}\mathbf{T} \\
\mathbf{S}\end{array}$ & $\mathrm{H}$ & \\
\hline $\begin{array}{l}4^{\text {th }} \text { and } \\
5 \text { th }\end{array}$ & 90.51 & $\begin{array}{l}\mathrm{C} \\
\mathrm{H}\end{array}$ & $\begin{array}{l}\mathrm{T} \\
\mathrm{D}\end{array}$ & $\begin{array}{l}\mathrm{T} \\
\mathrm{D}\end{array}$ & $\begin{array}{l}\text { D } \\
\mathbf{M}\end{array}$ & $\begin{array}{l}\mathbf{D} \\
\mathbf{M}\end{array}$ & $\mathrm{H}$ & \\
\hline $2 \mathrm{nd}$ & 90.51 & $\mathrm{H}$ & $\begin{array}{l}\text { D } \\
\text { M }\end{array}$ & $\begin{array}{l}\mathrm{C} \\
\mathrm{A}\end{array}$ & $\begin{array}{l}\mathrm{C} \\
\mathrm{O} \\
\mathrm{A}\end{array}$ & $\mathrm{H}$ & $\begin{array}{l}\mathrm{T} \\
\mathrm{D}\end{array}$ & \\
\hline 5 th & 90.58 & $\begin{array}{l}\mathrm{T} \\
\mathrm{S}\end{array}$ & $\begin{array}{l}\text { M } \\
\mathrm{O} \\
\mathrm{O}\end{array}$ & W & $\begin{array}{l}\text { M } \\
\mathrm{O} \\
\mathrm{E}\end{array}$ & $\begin{array}{l}\text { D } \\
\text { F }\end{array}$ & $\begin{array}{l}\mathrm{C} \\
\mathrm{I}\end{array}$ & \\
\hline 6 th & 90.42 & $\begin{array}{l}\mathrm{C} \\
\mathrm{A}\end{array}$ & $\begin{array}{l}\mathrm{T} \\
\mathrm{D}\end{array}$ & $\mathrm{H}$ & $\begin{array}{l}\mathrm{C} \\
\mathrm{A}\end{array}$ & $\begin{array}{l}\mathrm{T} \\
\mathrm{D}\end{array}$ & $\begin{array}{l}\text { D } \\
\text { M }\end{array}$ & \\
\hline 5 th & 90.29 & $\begin{array}{l}\mathrm{C} \\
\mathrm{A}\end{array}$ & $\begin{array}{l}\mathrm{C} \\
\mathrm{M} \\
\mathrm{M}\end{array}$ & $\begin{array}{l}\mathrm{T} \\
\mathrm{S}\end{array}$ & $\mathrm{H}$ & $\begin{array}{l}\text { D } \\
\mathbf{M}\end{array}$ & $\mathrm{H}$ & \\
\hline $\begin{array}{l}\text { 3rd and } \\
5 \text { th }\end{array}$ & 90.24 & $\begin{array}{l}\mathrm{C} \\
\mathrm{A}\end{array}$ & $\mathrm{H}$ & $\begin{array}{l}\text { D } \\
\text { M }\end{array}$ & $\begin{array}{l}\mathrm{T} \\
\mathrm{S}\end{array}$ & $\begin{array}{l}\text { D } \\
\text { M }\end{array}$ & $\mathrm{H}$ & \\
\hline 4th & 91.64 & $\begin{array}{l}\mathrm{C} \\
\mathrm{A}\end{array}$ & $\begin{array}{l}\mathrm{T} \\
\mathrm{S}\end{array}$ & $\mathrm{H}$ & $\begin{array}{l}\text { D } \\
\text { M }\end{array}$ & $\begin{array}{l}\mathrm{M} \\
\mathrm{O} \\
\mathrm{O}\end{array}$ & & \\
\hline 4th & 90.30 & $\mathrm{~T}$ & $\mathrm{H}$ & $\mathrm{C}$ & D & $\mathrm{T}$ & & \\
\hline
\end{tabular}




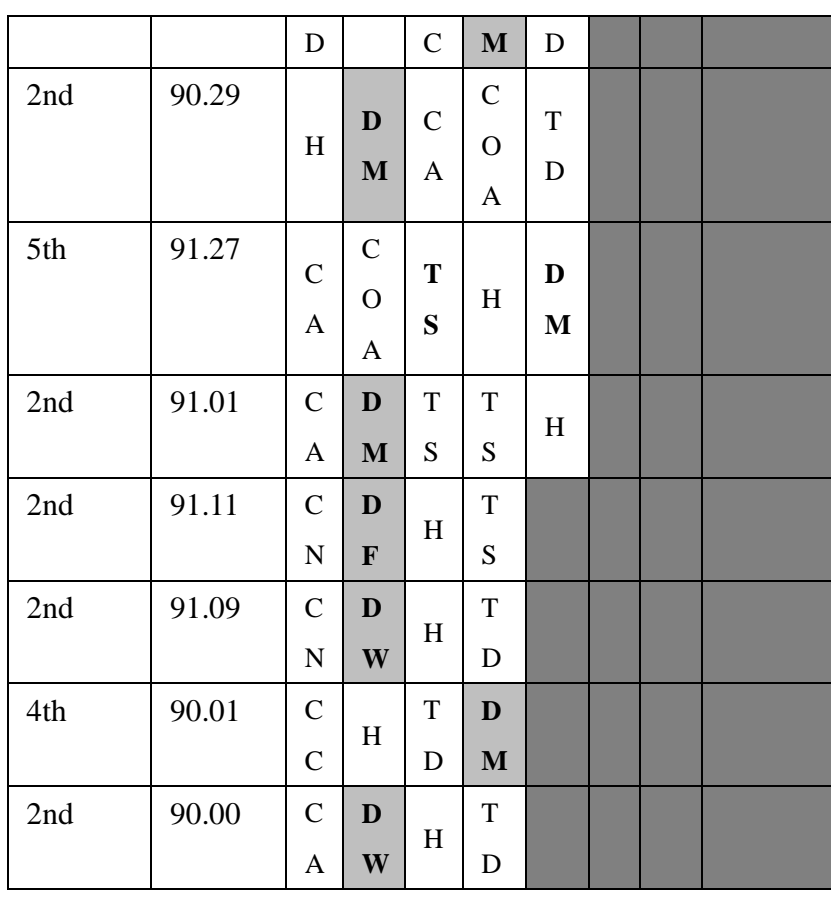

Legends

\begin{tabular}{|l|l|l|}
\hline CA - Contrast & $\begin{array}{l}\text { MOO - Morphological } \\
\text { Enhancement }\end{array}$ & $\begin{array}{l}\text { CMM - Combine } \\
\text { Adapthisteq }\end{array}$ \\
CN - Contrast & DM - Denoise Median & COA - Combine \\
Enhancement NBins & DW - Denoise Wiener & Average \\
CC - Contrast & DF - Denoise ImFilter & COM - Combine \\
Enhancement ClipLimit & TS - Thresholding & Multiply \\
CI-Contrast ImAdjust & Simple & H - Hole Filling \\
CH - Contrast Histeq & TD - Thresholding & W- Watershed \\
MOE - Morphological & & \\
Operator Eroding & & \\
\hline
\end{tabular}

As for Table 3, it shows IPCO chains with an F1 score larger than $90 \%$, averaged over all training images, with legends describing the abbreviations used in the table. In comparison with the results in Table 2, the optimizer tends to choose a larger variety of Denoising functions (e.g. Wiener and Imfilter (MatLab built in function)). The same applies to Contrast Enhancement, where the optimizer tends to also choose Global Contrast functions such as ImAdjust and Histeq (Histogram Equalization), on top of other Local Contrast Enhancement functions such as NBins and ClipLimit. As for Thresholding, it also exhibits a larger variety of selected functions, including Double Thresholding.
According to Table 3, which depicts 30 IPCO chains with F1 scores larger than $90 \%, 43 \%$ of chains have Denoising in second place, $13 \%$ in third place, $23 \%$ in fourth place, $30 \%$ in fifth place, $20 \%$ in sixth place, and $6 \%$ in eighth place. Fig. 3 depicts a graphical representation of the information in Table 3 pertaining to the percentage of chains with Denoising at different relative chain positions.

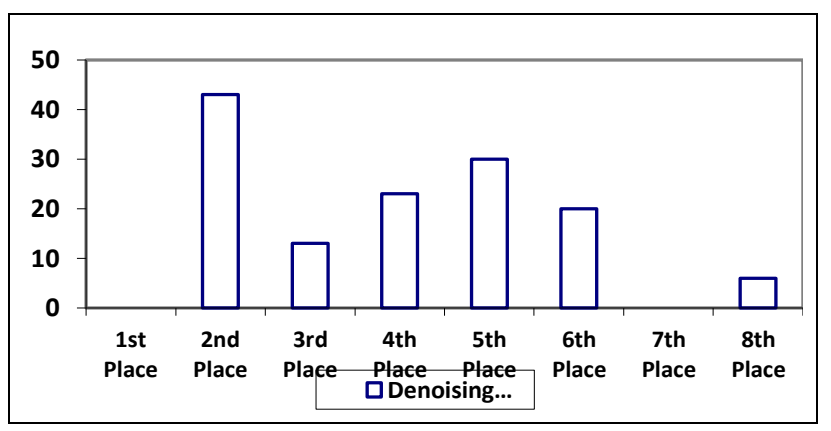

Fig. 3. Percentage of chains with Denoising at different relative chain positions

\subsubsection{Shortest chains}

The shortest chain with an F1 score larger than $91 \%$ consists of 4 functions, with Denoising in the second position of the chain. The chain begins with Contrast Enhancement followed by Denoising, followed by Hole-filling and finally Thresholding. Another interesting (small) chain of length 5 (i.e. using 5 functions), adopts Denoising at the final stage. From the first to the last function, the chain consists of: Contrast Enhancement, Average, Thresholding, Hole-filling and Denoising. Another example of Denoising as a final component consists of a chain of length 8 , which actually employs Denoising 3 times.

\subsubsection{Best Single Chain}

From our experiments, if we classify each function in terms of its general purpose (i.e. enhancement, classification and cleaning) we arguably obtain the following simplified chains:

- $\quad$ First Best Chain:

Enhance $\rightarrow$ Classify $\rightarrow$ Clean $\rightarrow$ Clean $\rightarrow$ Classify $\rightarrow$

Classify $\rightarrow$ Clean

- $\quad$ Second Best Chain:

Enhance $\rightarrow$ Classify $\rightarrow$ Classify $\rightarrow$ Clean $\rightarrow$ Clean 
Table 1 depicts image processing phases with their corresponding general purposes. The first and second best chains were taken from Table 2 . The chains have average F1 scores of $91.67 \%$ and $91.64 \%$ respectively. Below we explicitly show the matching between processing function and purpose.

- $\quad$ First Best Chain: Enhance (Contrast Enhancement) $\rightarrow$ Classify (Thresholding) $\rightarrow$ Clean ( Morphological Operator) $\rightarrow$ Clean (Denoising) $\rightarrow$ Classify (Watershed) $\rightarrow$ Classify (Hole Fill) $\rightarrow$ Clean (Morphological Operator)

- $\quad$ Second Best Chain: Enhance (Contrast Enhancement) $\rightarrow$ Classify ( Thresholding) $\rightarrow$ Classify ( Hole Fill) $\rightarrow$ Clean (Denoising) $\rightarrow$ Clean ( Morphological Operator)

As we can see, in both chains, cleaning only takes place after enhancing and classification. This arguably runs contrary to common expectation. This is something that needs to be taken into account by image processing users, that we should not always clean images at an early stage since this will remove important information that may be needed by other component functions.

\section{Conclusions}

From our experiments, and given the specific membrane detection dataset adopted, we find that the optimization of image processing chains, suggests that details need to be enhanced before cleaned. In fact the results even suggest that classification is often best done before Denoising. This arguably runs contrary to common expectation and this insight can probably be best encapsulated by the heuristic "enhance it before you lose it". Although typically in image processing, Denoising is adopted as a first (or early) pre-processing step, this work serves as clear warning that this choice may not always be optimal. This is likely to be true for many applications, where noise needs to be protected in the early stages such as in the field of medical imaging where every detail is likely to be important from the clinical (e.g. diagnostic) point of view. In our experiments, we have found that the best scoring image processing chains perform both image enhancement and classification before Denoising.

\section{Acknowledgment}

We would like to thank ISBI (International Symposium on Biomedical Imaging) for providing the training, test and ground-truth datasets.

\section{References}

(1) Raju,R.,Maul,T.H.,Bargiela,A : "Local Contrast Hole Filling Algorithm", IEEE Symposium on Computer Applications \& Industrial Electronics, ISCAIE, 2014

(2) Rafael C.Gonzales, Richard E.Woods, Steven L.Eddins, : "Digital Image Processing using MatLab", McGraw-Hill, 2010

(3) Reiner Hegerl and Achilleas S. Frangakis : "Denoising of Electron Tomograms", Manuscript, Chapter 11, pp 331 to 351,2006

(4) R.Sivakumar : "Denoising Of Computer Tomography Images Using Curvelet Transform", ARPN Journal of Engineering and Applied Sciences, Vol. 2, No. 1, pp. 21-26, 2007

(5) A. Buades, B. Coll, and J. Morel : "Neighborhood Filters and PDE's", Technical Report 2005-04, CMLA, 2005

(6) Priyam Chatterjee and Peyman Milanfar : "Is Denoising Dead?", IEEE Transactions On Image Processing, Vol 19, No 4, 2010

(7) Alexander Wong, Akshaya Mishra, Wen Zhang, Paul Fieguth, and David A. Clausi : "Stochastic Image Denoising Based on Markov-Chain Monte Carlo Sampling", Department of Systems Design Engineering, University of Waterloo, March 26, 2011

(8) D. Shattuck, S. Sandor-Leahy, K. Schaper, D. A. Rottenberg, and R. M. Leahy : "Magnetic resonance image tissue classification using a partial volume model", Neuroimage, Vol. 13, no. 5, pp. 856-876, 2001

(9) J. Ashburner and K. J. Friston : "Multimodal image coregistration and partitioning - a unified framework", NeuroImage, Vol. 6, no. 3, pp. 209-217, 1997

(10) J. C. Kosior, R. K. Kosior, and R. Frayne : "Robust dynamic susceptibility contrast MR perfusion using 4D nonlinear noise filters", Journal of Magnetic Resonance Imaging, Vol. 26, no. 6, pp. 1514-1522, 2007

(11) S. Annadurai, R. Shanmugalakshmi : "Fundamentals of Digital Image Processing", Pearson Education India, 2006

(12) Joao M. Sanches, Jacinto C. Nascimento and Jorge S. Marques : "Medical Image Noise Reduction Using the 
Sylvester-Lyapunov Equation", IEEE transactions on image processing, Vol. 17, No. 9, pp. 1522-1539, 2008

(13) Kenneth R.Spring, John C.Russ, Micheal W.Davidson : "Recommended Strategy for Processing Digital Images”, Olympus America Inc, 2012

(14) Nilamani Bhoi and Sukadev Meher: "Total Variation Based Wavelet Domain Filter for Image Denoising", In proceedings of the First International Conference on Emerging Trends in Engineering and Technology, pp. 20-25, 2008

(15) Charandeep Singh Bedi and Himani Goyal : "Qualitative and Quantitative Evaluation of Image Denoising Techniques", International Journal of Computer Application, 2010

(16) ISBI 2012 challenge, http://bit.ly/riGDUm

(17) Cardona, A., Saalfeld, S., Preibisch, S., Schmid, B., Pulokas, A.C.J., Tomancak,P., Hartenstein, V : “An integrated micro- and macro architectural analysis of the drosophila brain by computer-assisted serial section electron microscopy", 2010

\section{Authors and Affiliations}

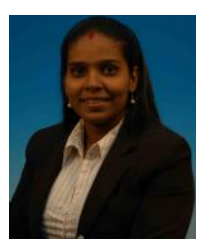

Rajeswari Raju is currently doing $\mathrm{PhD}$ in the School of Computer Science at the University of Nottingham, Malaysia Campus. She received her Master Degree in Computer Science and Bach (Hons) Degree in Information Technology from University Malaya. She is a Lecturer at University Technology MARA (UiTM) Terengganu Campus.

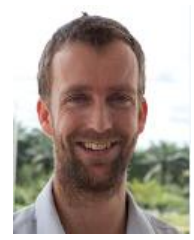

Tomás H. Maul received the B.Sc. (Hons.) degree in biological psychology from the University of St. Andrews, St. Andrews, U.K., the M.S. degree in computer science from Imperial College, London, U.K., and the Ph.D. degree in computational neuroscience from the University of Malaya, Kuala Lumpur, Malaysia. For two years, he was a Senior Researcher with MIMOS Berhad, where he worked in the fields of pattern recognition and computer vision. He is currently an Assistant Professor with The University of Nottingham Malaysia Campus, Semenyih, Malaysia, where he conducts research in the areas of neural computation and computer vision.

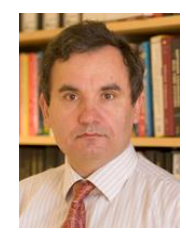

Andrzej Bargiela is Professor in the School of Computer Science at the University of Nottingham and Institute of Informatics at Krakow University of Technology. He served as President of the European Council for Modelling and Simulation (ECMS) during 2002-2006 and 2010-2012. He is Associate Editor of the IEEE Transactions on Systems Man and Cybernetics and Associate Editor of the Information Sciences. His research involves investigation into Granular Computing, human-centred information processing as a methodological approach to solving large-scale data mining. 